\title{
Does a positive Heaf test reaction in Asian schoolchildren predict later breakdown of tuberculosis?
}

\author{
J B Cookson, A G I Cookson
}

\begin{abstract}
Background Some authorities advise giving prophylactic antituberculosis treatment to those with a grade 3 or 4 reaction to Heaf tests performed as part of the schools' BCG programme. This is not done in Leicestershire, which because of its large Asian population would require considerable resources to do so. A calculation of the "breakdown" rate, and thus the potential value of chemoprophylaxis, in these children would therefore be valuable.

Method Lists of names of Asian children who had a grade 3 or 4 Heaf test reaction in 1982 and 1983 were compared with lists of notifications for the five years 1983 to 1987. Those who were on both lists were considered to have tuberculosis that had "broken down."

Results Of the 760 children with positive Heaf test reactions, only four were on the subsequent notification lists. The chest radiograph obtained because of the Heaf test result showed evidence of active tuberculosis in only three of these. Only one child had infection that "broke down" later.

Conclusion The benefit of chemoprophylaxis seens likely to be limited in these Asian children, most of whom had received BCG early in life.
\end{abstract}

(Thorax 1992;47:776-777)

The Department of Health advise that schoolchildren who show strongly positive tuberculin reactions when routinely tested under the BCG vaccination programme at around the age of 13 should be referred for further investigation and supervision (which may include prophylactic chemotherapy). ${ }^{12}$ Current policy in Leicestershire is to perform Heaf tests on schoolchildren at the age of 12-13 years. Those with strongly positive reactions (grades 3 and 4 ) have chest radiographs taken, but if these are normal then no action is taken and no chemoprophylaxis is given. There is a risk that some of these will "break down" and develop clinical tuberculosis, which might otherwise be prevented with isoniazid prophylaxis. Many children, however, may be treated unnecessarily. Immense clinic resources will be needed, and drug related side effects, though rare, are possible. Compliance may be low as patients will not feel "ill." It would therefore be helpful to know the "breakdown" rate in this population, to give some estimate of the likely benefit from prophylaxis.

The problem is particularly acute in Leicestershire with its large Asian community, and as there is little information about this population we decided to study only this group.

\section{Method}

Notification forms used by health visitors for contact tracing are a complete record of tuberculosis notifications in Leicestershire. These records for the five years 1983-7 were scanned for children with Asian sounding names born around 1970 and therefore aged around 12 or 13 in 1982 and 1983

These names of children with active tuberculosis were then compared with the lists of those children who had a grade 3 or 4 Heaf test reaction in 1982 and 1983. Dates of birth and addresses were compared as a further check because of the similarity of many Asian names. From these figures a "breakdown" rate was calculated.

\section{Results}

Tuberculosis notifications in all groups in Leicestershire for the years 1983-7 ranged from 159 to 241 /year.

The number of Asian schoolchildren with a grade 3 or 4 reaction to Heaf tests performed as part of the BCG vaccination programme in 1982 and 1983 was 760 .

The number of tuberculosis notifications in Asian children in the years 1983-7 who were documented as having a grade 3 or 4 Heaf test reaction in 1982-3 was four. The initial chest radiograph, obtained because of the positive result, was thought to indicate active tuberculosis in three of the four children. Only one developed tuberculosis later.

\section{Discussion}

This study suggests that prophylaxis in this population is unlikely to have a major effect on later tuberculosis notifications. It would need to be given to 760 children, according to our results, to prevent one case in five years.

Possibly some children Heaf tested outside the usual age range were missed but care was taken to look beyond the age range 12-13 in the notification data to pick up all who could have been tested in the years 1982-3. Migration out of the area is a possible confounding factor. We are unable to obtain information on this cohort but more recent, computerised, data held by 
the Leicestershire Family Health Services Authority suggests that movement out of Leicestershire by Asian children about this age accounts for around $1 \%$ of the population each year. Cases may not be notified but we believe this to be rare; contact tracing is organised centrally by a single chest physician.

As the years pass, an increasing proportion of Asian children are born in the United Kingdom. This factor, and BCG programmes in the UK and abroad, may influence tuberculosis rates. In a separate (unpublished) study of 82 Asians born around 1972 (that is, they were two years younger than the present cohort) with grade 3 or 4 Heaf test results in the schools' BCG programme, we found that $80 \%$ had evidence of a BCG scar and $60 \%$ had been born in the UK. In the group of Asian schoolchildren in the present study therefore about $80 \%$ are likely to have received BCG vaccination early in life. This protection, reflecting the neonatal BCG vaccination rate among Asians in Leicester, may be a factor in our results, which may not therefore be applicable to other populations.

Our follow up period was five years. Possibly more cases of breakdown of tuberculosis would have been found had the follow up been longer. Prophylaxis should prevent breakdown for life. Late breakdown may undoubtedly occur but contact studies $^{3}$ suggest that most clinical disease occurs within two years of infection.

In Edinburgh 89590 (mostly white) children in the schools' programme were followed for a mean of seven years. ${ }^{4}$ Of 390 children with grade 3 or 4 Heaf test teactions, 13 were subsequently notified as having tuberculosis. Nine of these had an initial abnormal chest radiograph, leaving four (about one in 100) children tested who might benefit from prophylaxis. This might be considered worth while, especially as only $5 \%$ of the whole population had received BCG when younger.

A British Thoracic Association survey ${ }^{5}$ on the efficacy of BCG vaccination showed an annual breakdown rate in those with a positive Heaf test reaction of $22 / 100000$, but this included all grades.

Ormerod $^{6}$ showed that the introduction of a policy of prophylactic chemotherapy was associated with reduced notification rates in children, but he included contacts of cases, new immigrants, and recent Heaf converters as well as those detected by routine testing. The decline he showed could be due to the fact that later cohorts of children were born in the UK and received BCG vaccination at birth, as recommended by the Department of Health.

The recent code of practice of the British Thoracic Society ${ }^{7}$ on tuberculosis control recommends that tuberculin positive children who are new immigrants or recent contacts should receive prophylaxis but does not consider the schools' BCG programme. Advice from the same body ${ }^{8}$ on the management of tuberculosis is to give prophylaxis to children with strongly positive Heaf test reactions but it does not qualify this advice.

Tuberculosis in small children is a potentially most damaging disease and therefore prevention would be worth while. Prophylaxis at 13 , however, prevents disease only in adolescents and adults. Our conclusion is that routine prophylaxis is not justified for this group of Asian children with strongly positive tuberculin test reactions, most of whom have received BCG vaccine in early life, so long as the chest radiographs are clear. Resources should be concentrated on early detection of tuberculosis in patients with symptoms.

1 Department of Health. Immunisation against infectious disease: London: HMSO, 1990.

2 Department of Health and Social Security. Tuberculosis epidemiology and control. London: DHSS, 1973.

3 British Thoracic Association. A study of a standardised contact procedure in tuberculosis. Tubercle 1978;19:245.

4 Capewell S, France A, Uzel N, Leitch G. The current value of tuberculin testing and BCG vaccination in schoolchildren. Br J Dis Chest 1986;80:254-64.

5 British Thoracic Association. Effectiveness of BCG vaccination in Great Britain in 1978. Br J Dis Chest 1980;74: 215-27.

6 Ormerod LP. Reduced incidence of tuberculosis by prophylactic chemotherapy in subjects showing strong reactions to tuberculin testing. Arch Dis Child 1987;62:1005-8.

7 Subcommittee of the Joint Tuberculosis Committee of the British Thoracic Society. Control and prevention of tuberculosis in Britain: an updated code of practice. BMJ 1990;300:995-9.

8 Ormerod LP. Chemotherapy and management of tuberculosis in the United Kingdom: recommendations of the Joint Tuberculosis Committee of the British Thoracic Society. Thorax 1990;45:403-8. 